\title{
Preventive Community Trial
}

National Cancer Institute

\section{Source}

National Cancer Institute. Preventive Community Trial. NCI Thesaurus. Code C39497.

The Preventive Community Trial is a controlled study that involves administration of preventive program or/and preventive treatments in community. 